\title{
Relaxation Dynamics and Cold Crystallization of Poly(pentamethylene terephthalate) as Revealed by Dielectric Spectroscopy
}

\author{
M. Soccio ${ }^{a^{*}}$, A. Nogales $^{a}$, I. Martín-Fabiani $^{\mathrm{a}}$, N. Lotti ${ }^{\mathrm{b}}$, A. Munari ${ }^{\mathrm{b}}$, T.A. Ezquerra ${ }^{\mathrm{a}^{*}}$ \\ ${ }^{a}$ Instituto de Estructura de la Materia, IEM-CSIC, Serrano 119, Madrid 28006, Spain \\ ${ }^{b}$ Dipartimento di Ingegneria Civile, Chimica, Ambientale e dei Materiali, Universitá di \\ Bologna, Via Terracini 28, Bologna 40131, Italy
}

\begin{abstract}
The relaxation dynamics of poly(pentamethylene terephthalate) has been investigated by means of dielectric spectroscopy. The sub-glass dynamics is characterized by the existence of a bimodal $\beta$ process whose faster and slower components have been assigned to the relaxation of the bond between the ester oxygen and the aliphatic carbon and to the link between the aromatic ring carbon and the ester carbon, respectively. By comparison with other closely related aromatic polyesters it is shown that the faster component strongly depends on the amount of methylene groups while the slower one is not considerably affected by the nature of the glycol subunit. The changes in the $\alpha$ process associated to the segmental relaxation during cold crystallization reveal the formation of a rigid amorphous phase fraction. Combination of dielectric experiments with X-ray scattering ones suggests that during cold crystallization PPT crystal lamellae tend to fill the space homogeneously.
\end{abstract}

Keywords: Poly(pentamethylene terephthalate); Dielectric Spectroscopy; Dynamics; Cold Crystallization

* Corresponding authors. Instituto de Estructura de la Materia, IEM-CSIC, Serrano 119, Madrid 28006, Spain.

Tel. (0034) 915616800 - 942412

E-mail addresses: m.soccio@iem.cfmac.csic.es (M. Soccio), t.ezquerra@csic.es (T.A. Ezquerra). 


\section{Introduction}

Poly(pentamethylene terephthalate) (PPT) is a semicrystalline aromatic polyester, not yet industrially available, belonging to the same family of other more studied terephthalic polyesters such as poly(ethylene terephthalate) PET, poly(trimethylene terephthalate) PTT and poly(butylene terephthalate) $\mathrm{PBT}^{1}$. Taking into account the outstanding properties of these polymers, PPT could be also a promising engineering thermoplastic material. An interesting difference with respect to the best known terephthalic polyesters, is its lower glass transition temperature, $T_{\mathrm{g}}$, below room temperature. This can make semicrystalline PPT to have lower strength and rigidity leading, among others, to a better impact resistance at room temperature and above. It is well established that the final properties of semicrystalline polymers directly depend on the structure, the morphology, and the relaxation dynamics of the material. Crystallographic studies on PET have shown that it crystallizes with the chain in a fully extended conformation while PTT, PBT and PPT crystallize with the chain contracted ${ }^{2}$. Crystallographic data on higher members of the series from poly(hepta methylene terphthalate) until poly( deca-methylene terephthalate $)^{3}$ indicates that while the odd members are contracted, the even members are extended. Although several works related to the crystallization and morphology of PPT have been published ${ }^{2,4-6}$ less attention has been devoted to the study of the relaxation dynamics of PPT. Dielectric spectroscopy (DS) is a technique especially suited to investigate the dynamics in polymers. The segmental mobility is revealed in DS experiments by the $\alpha$ relaxation while the $\beta$ relaxation is associated to the local dynamics ${ }^{7-10}$. In this respect it is noteworthy to remark the influence of the crystalline phase on the segmental dynamics of the amorphous regions in semicrystalline polymers. Most commonly, during polymer crystallization, the segmental relaxation ( $\alpha$ process) becomes less intense, broader, and slower as compared with that of the analogous amorphous polymer ${ }^{10-15}$. By monitoring these changes during crystallization, one can extract both dynamical and structural information on the amorphous fraction hardly attainable by other techniques. Generally the semicrystalline state of most crystallizable polymers cannot be simply described by means of a two-phase model consisting of crystalline and amorphous phases. Consequently, a third phase, referred to as "rigid-amorphous phase" (RAP) has to be considered $^{16,17}$. The RAP can be defined as that portion of material that even though non-crystalline is not able to relax at the same rate as the mobile amorphous fraction. DS measurements have evidenced the existence of a rigid amorphous phase in different 
polymers including $\mathrm{PET}^{11,18}, \mathrm{PTT}^{19,20}$ and $\mathrm{PBT}^{17}$. Moreover, simultaneous DS and Xray diffraction experiments during isothermal crystallization close above $T_{g}$ (cold crystallization) support that while PET exhibits an heterogeneous distribution of crystalline lamellar stacks $^{11}$, PTT shows and homogeneous filling of lamellar crystals ${ }^{20}$. Considering that PBT is not suitable for these studies since cannot be obtained amorphous at room temperature then, it is clear that a detailed study of PPT can be of interest as a complement of the previous data in order to get a better description of the interrelation between structure and dynamics in aromatic polyesters. This work presents experimental results on the relaxation behavior of PPT as revealed by dielectric spectroscopy in order to provide information on both local and segmental dynamics in this aromatic polyester. Moreover isothermal cold crystallization, has been studied both by dielectric spectroscopy and wide-angle X-ray scattering (WAXS), to shed light on the structure-dynamics relationships of PPT and compare these with other semicrystalline aromatic polyesters.

\section{Experimental part}

\subsection{Samples.}

Poly(pentamethylene terephthalate) (PPT) was synthesized according to the two-stage polycondensation procedure ${ }^{21}$, starting from 1-5 pentanediol (PD) and dimethylterephthalate (DMT) with a molar ratio PD/DMT=1.7/1, employing titanium tetrabutoxide as catalyst (about $0.2 \mathrm{~g}$ of $\mathrm{Ti}(\mathrm{OBu})_{4} / \mathrm{kg}$ of polymer). The synthesis was carried out in a $200 \mathrm{~mL}$ stirred glass reactor, with a thermostatic silicon oil bath; temperature and torque were continuously recorded during the polymerization. In the first stage, under pure argon flow, the temperature was raised to $230{ }^{\circ} \mathrm{C}$ and maintained there until more than $90 \%$ of the theoretical amount of methanol was distilled off (about $2 \mathrm{~h}$ ). In the second stage, the pressure was reduced to facilitate the removal of the glycol in excess and the temperature was raised to $250{ }^{\circ} \mathrm{C}$ and maintained there until a torque constant value was measured. The chemical structure, presented in Figure 1, was corroborated by means of ${ }^{1} \mathrm{H}-\mathrm{NMR}$ spectroscopy (Varian INOVA $400 \mathrm{MHz}$ instrument). The chemical shift assignments $(\delta, \mathrm{ppm})$ are the following: $8.16\left(4 \mathrm{H}^{\mathrm{a}}\right), 4.43$ $\left(4 \mathrm{H}^{\mathrm{b}}\right), 1.95\left(4 \mathrm{H}^{\mathrm{c}}\right), 1.65\left(2 \mathrm{H}^{\mathrm{d}}\right)$ (see Figure 1). The polymer was obtained with a molecular weight $M_{n}=54000 \mathrm{~g} / \mathrm{mol}$ and a polydispersity $D=2.2$. The as prepared polymer is semicrystalline with a calorimetric glass transition temperature $\left(T_{g}\right)$ of $16{ }^{\circ} \mathrm{C}$ and a melting temperature $\left(T_{m}\right)$ of $129^{\circ} \mathrm{C}$. 


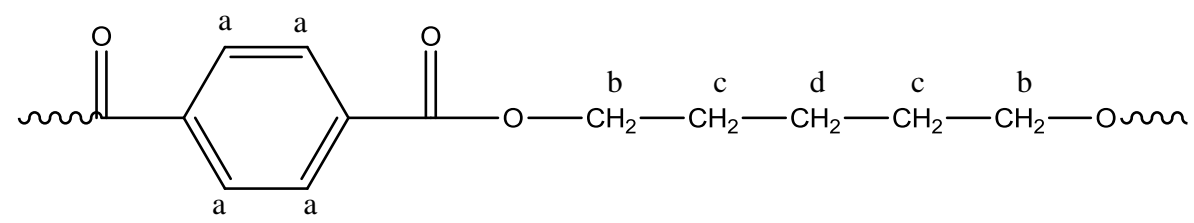

Figure 1. Chemical structure of the repetitive unit of poly(pentamethylene terephthalate). Labels indicate the atoms giving rise to ${ }^{1} \mathrm{H}-\mathrm{NMR}$ chemical shift signals.

PPT amorphous films can be prepared as follows: after vacuum drying at $25{ }^{\circ} \mathrm{C}$ for 24 $\mathrm{h}$, the powders originated from the synthesis were melt pressed at $160{ }^{\circ} \mathrm{C}$ for $4 \mathrm{~min}$ and subsequently quenched using iced water and kept below the calorimetric $T_{g}\left(16^{\circ} \mathrm{C}\right)$ to avoid the crystallization process. In that way polymer amorphous films of about $250 \mu \mathrm{m}$ thick were obtained.

2.2. Broadband Dielectric Spectroscopy (DS). Complex dielectric permittivity measurements $\left(\varepsilon^{*}=\varepsilon^{\prime}-i \varepsilon^{\prime \prime}\right)$ were performed over a $10^{-1}<F / H z<10^{6}$ frequency range in a temperature range of $-150{ }^{\circ} \mathrm{C}<T<+125^{\circ} \mathrm{C}$. A Novocontrol system integrating an ALPHA dielectric interface was employed. The temperature was controlled by means of a nitrogen gas jet (QUATRO from Novocontrol) with a temperature error of $\pm 0.1^{\circ} \mathrm{C}$ during every single sweep in frequency. PPT films were sandwiched between the two metallic electrodes of the spectrometer. Due to the low glass temperature of PPT no gold evaporated/sputtered electrodes were used in order to avoid crystallization induced by the deposition. In order to characterize the influence of crystallization in both local and segmental relaxation two different types of DS experiments were performed. In the first one, PPT amorphous film was isothermally crystallized inside the Novocontrol spectrometer, at $30^{\circ} \mathrm{C}$, with a time interval between dielectric measurements of $10 \mathrm{~min}$. At this temperature the $\alpha$ process appears well centred within the frequency range. In the second type of experiment, firstly the sample was cooled down to $-85^{\circ} \mathrm{C}$ at $5^{\circ} \mathrm{C} / \mathrm{min}$ and a dielectric measurement was performed. At this low temperature the $\beta$ relaxation can be well characterized and the crystallization process is in standby because the temperature is well below $T_{\mathrm{g}}$. Subsequently, the sample was heated up to $\mathrm{T}=30^{\circ} \mathrm{C}>T_{\mathrm{g}}$ in order to perform another frequency sweep, and held at this temperature for a controlled period. This procedure was repeated several times until crystallization was estimated to stabilize. Every single sweep in frequency lasted $2 \mathrm{~min}$. For the crystallization experiments with DS the samples was melt pressed between two metallic 
electrodes using Kapton $\bigcirc$ spacers in order to keep the thickness of the sample to a fix value $^{22}$.

2.3. X-ray Scattering (WAXS). Wide-angle X-ray scattering measurements as a function of time during isothermal crystallization at $30{ }^{\circ} \mathrm{C}$ were performed by means of a Nanostar equipment from Bruker using Ni-filtered $\mathrm{Cu} K \alpha$ wavelength $(\lambda=1.542 \AA)$. The experiment was performed in transmission geometry. The sample to detector distance was set to $10 \mathrm{~cm}$. The crystallinity index $\left(X_{\mathrm{c}}\right)$ was calculated as the ratio between the integrated area below the deconvoluted crystalline peaks to the total

experimental scattered integral intensity ${ }^{23,24}$. The contribution of the amorphous halo was taken from the initial pattern (crystallization time $\mathrm{t}_{\mathrm{c}}=0$ ).

\section{Results and discussion.}

\subsection{Dielectric spectroscopy of initially amorphous PPT}

Figure 2 shows the dielectric loss values as a function of temperature and frequency for initially amorphous PPT. The relaxation processes appear as maxima in $\varepsilon^{\prime \prime}$ versus frequency whose frequency of maximum loss moves toward higher values as temperature increases. Below the glass transition temperature, a broad maximum of the dielectric loss, labelled as $\beta$ process, appears. At temperatures above the $T_{\mathrm{g}}$ a strong increase in $\varepsilon^{\prime \prime}$ values, labelled as $\alpha$ process, is observed. Slightly above $T_{g}$ the $\alpha$ relaxation undergoes a sudden decrease in $\varepsilon^{\prime \prime}$. This effect is typical of a polymer that crystallizes at $\mathrm{T}>T_{\mathrm{g}}$ during the dielectric $\operatorname{scan}^{20}$. Therefore, during the dielectric run of the initially amorphous polymer we can distinguish two $\alpha$ relaxation processes. The first one related to the sample before crystallization $(\alpha)$ and a second one related to the polymer after crystallization $\left(\alpha^{\prime}\right)$. 


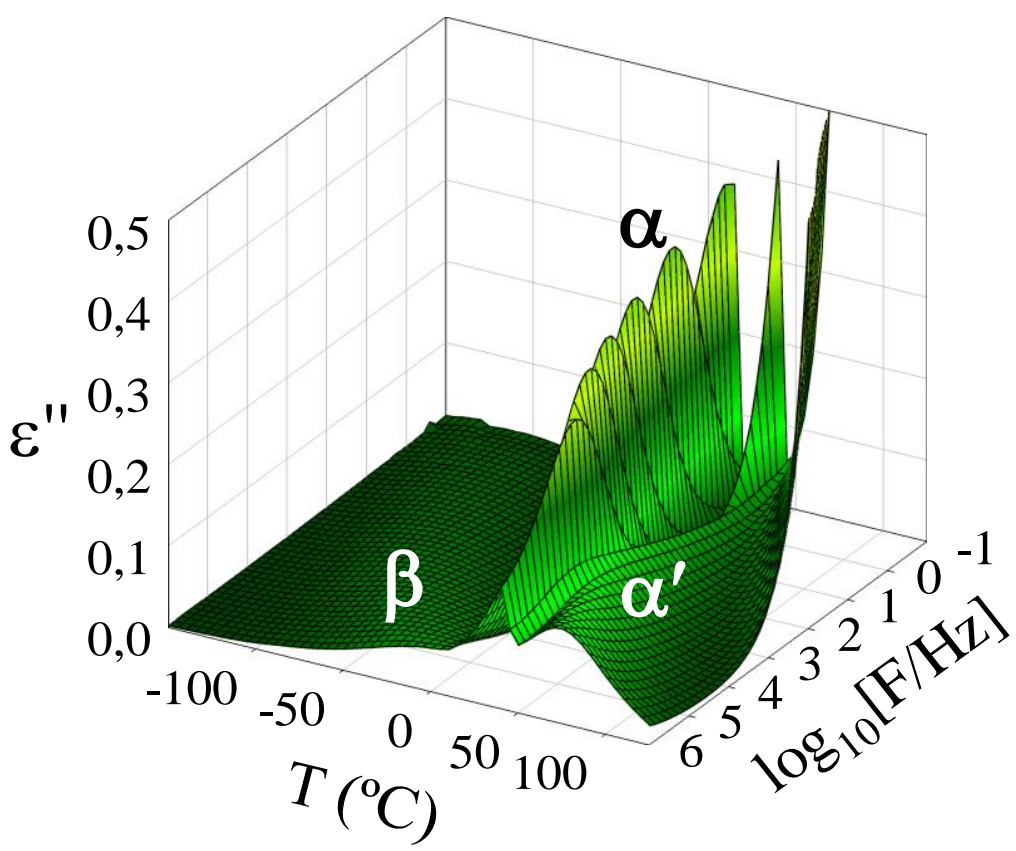

Figure 2. Dielectric loss values $\left(\varepsilon^{\prime \prime}\right)$ as a function of temperature and frequency for initially amorphous PPT.

\subsubsection{The $\beta$ relaxation of amorphous PPT}

The $\beta$ relaxation process observed below the $T_{\mathrm{g}}$ as a broad maximum in Figure 2 looks very similar to those observed in other aromatic polyesters ${ }^{10,19,20}$. Accordingly, it can be related to the local chain dynamics. Figure 3 shows, for three given temperatures, the $\beta$ relaxation of amorphous PPT. Close inspection of the $\beta$ relaxation reveals that it is rather broad and clearly composed of two processes, designated as $\beta_{2}$ and $\beta_{1}$ in order of increasing frequency. The slowest $\beta$ process $\left(\beta_{2}\right)$ shows smaller intensity as compare to the $\beta_{1}$ one.
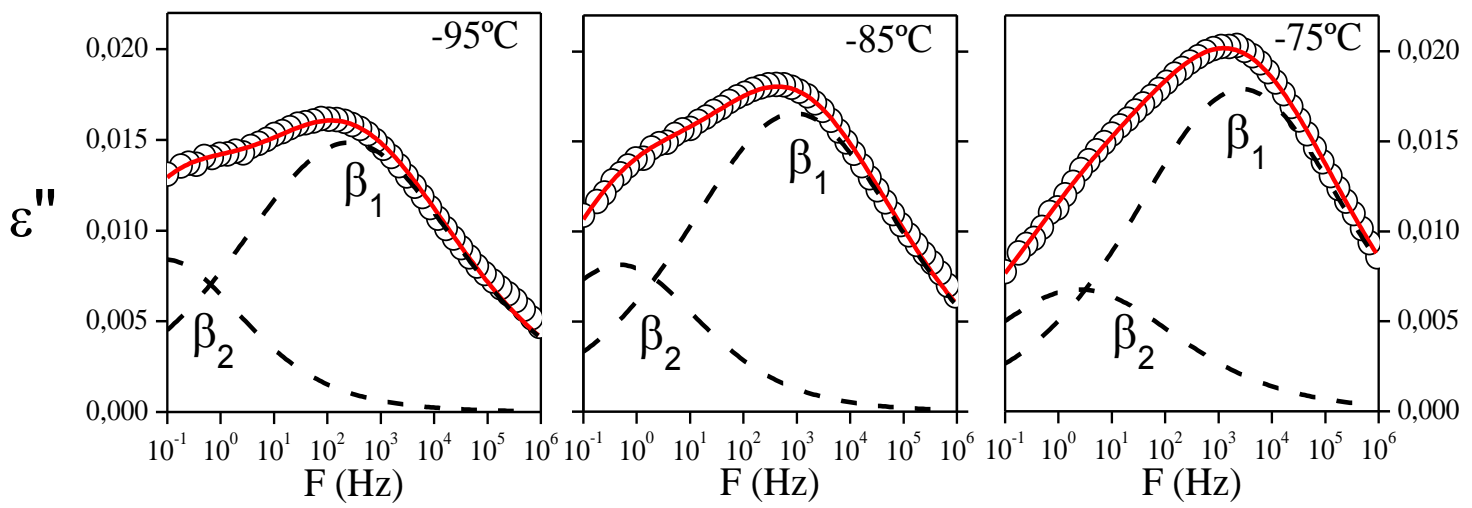

Figure 3. Isothermal $\varepsilon^{\prime \prime}$ data of initially amorphous PPT, at different temperatures. Continuous lines represent best fits according to CC equation, dashed lines show the separated contribution of the different relaxation processes. 
The dielectric relaxations can be described in general in terms of the Havriliak-Negami (HN) equation ${ }^{25}$ :

$$
\varepsilon^{*}=\varepsilon_{\infty}+\frac{\varepsilon_{0}-\varepsilon_{\infty}}{\left[1+(i \omega \tau)^{b}\right]^{c}}
$$

which describes the dependence of the complex dielectric permittivity, $\varepsilon^{*}$, with the angular frequency $\omega$. Here $\varepsilon_{0}$ and $\varepsilon_{\infty}$ are the relaxed $(\omega=0)$ and unrelaxed $(\omega=\infty)$ dielectric constant values, $\tau$ is the central relaxation time of the relaxation time distribution function, and $b$ and $c(0<b, c<1)$ are shape parameters which describe the symmetric and the asymmetric broadening of the relaxation time distribution function, respectively ${ }^{9}, 25$. The average relaxation time $\left(\tau_{\max }\right)$ value of the distribution can be calculated as follows 9 :

$$
\tau_{\max }=\frac{1}{2 \pi F_{\max }}=\tau_{H N}\left[\sin \frac{b \pi}{2+2 c}\right]^{-\frac{1}{b}}\left[\sin \frac{b c \pi}{2+2 c}\right]^{\frac{1}{b}}
$$

Where $F_{\max }$ is the frequency at which the maximum in dielectric loss appears, and $\tau_{\mathrm{HN}}$ is the central relaxation time of eq.1. For the temperature region of the $\beta$ relaxation, below $T_{\mathrm{g}}$, a satisfactory description of the experimental data can be achieved by assuming an sum of two process (eq.1) with symmetric shapes $(c=1)$. In this case eq. 1 is referred to as Cole-Cole (CC) equation ${ }^{9}$. The dotted lines in Figure 3 correspond to the contribution of the different $\beta_{1}$ and $\beta_{2}$ processes described by two independent CCprocesses. The continuous line is the total fit considering the additive contribution of both processes.

Figure 4 shows the $\tau_{\max }$ as a function of the reciprocal temperature for the $\beta$ relaxation processes. In this representation both $\beta$ components follow an Arrhenius behaviour as expected for sub-glass relaxation processes ${ }^{7}$. 


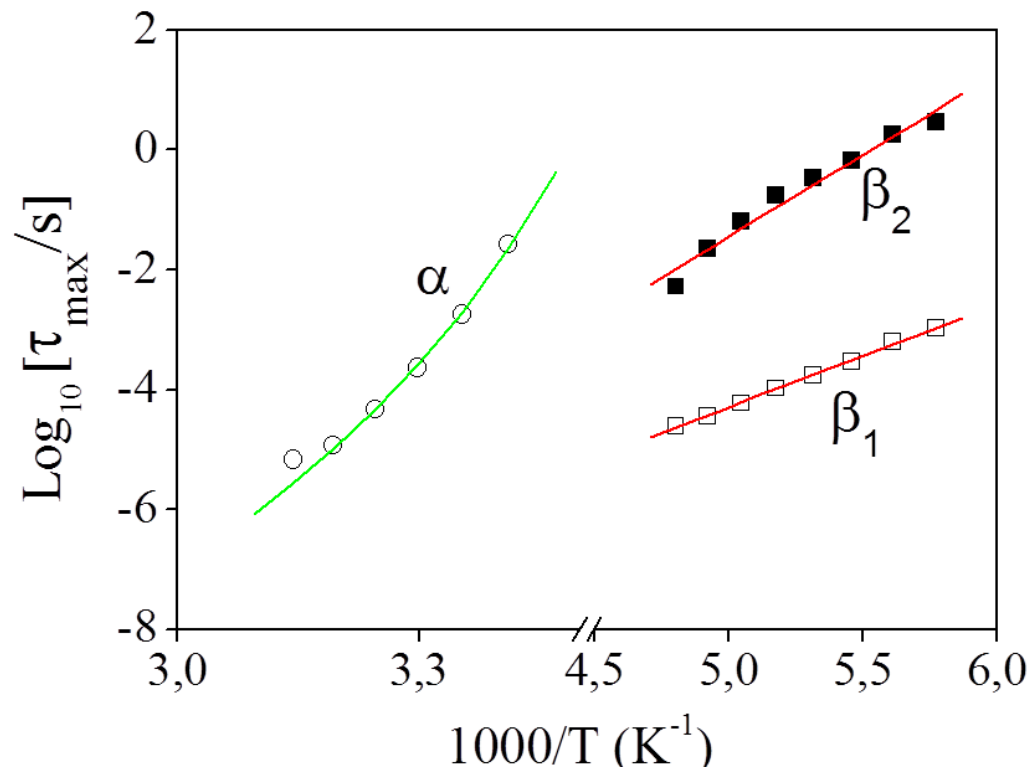

Figure 4. Logarithm of the average relaxation time as a function of the reciprocal temperature for amorphous PPT, for the local processes $\beta_{1}$ and $\beta_{2}$, and for the segmental relaxation $\alpha$. Continuous lines correspond to best fits to Arrhenius ( $\beta$ relaxations) and VFT equations ( $\alpha$ relaxation).

From the slope of the $\tau_{\max }$ it is possible to obtain the activation energy, $E_{\mathrm{a}}$. The $E_{\mathrm{a}}$ calculated for the $\beta_{1}$ and $\beta_{2}$ processes are $32 \mathrm{~kJ} \mathrm{~mol}^{-1}$ and $47 \mathrm{~kJ} \mathrm{~mol}^{-1}$, respectively. The $\beta$ relaxation in aromatic polyesters has been investigated in detail by molecular dynamics simulation $^{26}$. The multimodal shape of the $\beta$ relaxation in aromatic polyesters has been proposed to be a contribution of the three conformationally flexible bonds of the monomer, namely, the aromatic ring carbon to ester carbon bond (CA-C), the ester ether oxygen to aliphatic carbon bond $(\mathrm{O}-\mathrm{C})$ and the aliphatic carbon-carbon bond $(\mathrm{C}$ C). By modelling the conformational transition rates of these bonds it was reported that the O-C bond should relax faster than the $\mathrm{C}-\mathrm{C}$ one and both faster than the CA-C bond $^{26}$. This latter bond is responsible for the $\beta^{*}$ relaxation appearing in polyesters based on 2,6-naphthalenedicarboxylic acid like Poly(ethylene naphthalene-2,6dicarboxylate) $(\mathrm{PEN})^{27,28}$ or Poly(butylene naphthalene-2,6-dicarboxylate) $(\mathrm{PBN})^{29}$. In our case for PPT, the experimental dielectric data for the $\beta$ relaxation can be experimentally described by two components, $\beta_{1}$ and $\beta_{2}$. On the basis of the activation energy and on the previous discussion ${ }^{26,28}$, the molecular origin of the faster mode of the $\beta$ relaxation $\left(\beta_{1}\right)$ can be associated with the relaxation of the $\mathrm{O}-\mathrm{C}$ bond of the diol subunit. It is worth to mention that due to the small dipole moment of the $\mathrm{C}$-C bond, its relaxation is not likely to be detected by DS. Actually, polyolefins are not inherently 
dielectrically active and dielectric labelling through appropriate oxidation is needed in order to perform DS measurements ${ }^{30}$. In this respect we can associate the slowest component $\left(\beta_{2}\right)$ with the relaxation of the aromatic ring carbon to ester carbon bond (CA-C) although the influence of the $\mathrm{C}-\mathrm{C}$ bond to the $\beta_{2}$ component, if not through its dipole moment by its conformational motion, should not be discarded. In the present case for PPT the activation energy values are consistent with the above described assignment ${ }^{26,28}$. A multimodal shape of the glassy dynamics was already observed in other terephthalic acid containing polyesters like poly(ethylene terephthalate) $\mathrm{PET}^{28}$, poly(trimethylene terephthalate) $\mathrm{PTT}^{31}$, poly(butylene terephthalate) $\mathrm{PBT}^{32}$ among others $^{28,29}$. The aromatic polymers mentioned above differ by the number of methylene groups of the glycol subunit: 2, 3 and 4 for PET, PTT and PBT respectively. Table 1 reports the measured values of the activation energies of the $\beta_{1}$ and $\beta_{2}$ processes for different aromatic polyesters with varying number of $-\mathrm{CH}_{2}$ groups in their monomer glycol subunits. For the sake of comparison, the data for poly(neopenthyl terephthalate) (PNT) were also taken into account ${ }^{31}$. PNT has similar chemical structure than that of PTT but with two methyl groups instead of the two hydrogen atoms in $\beta$-position with respect to the oxygen atom.

Table 1. Activation energies $\left(E_{\mathrm{a}}\right)$ of the $\beta$ relaxations and $D$ and $T_{0}$ parameters of the $\alpha$ process, for amorphous PPT and other terephthalic acid containing amorphous polyesters: PET $^{33}$, PTT $^{31}$, poly(neopenthyl terephthalate) $(\mathrm{PNT})^{31}$. Data for semicrystalline $\mathrm{PBT}^{32}$ have been included for discussion.

\begin{tabular}{|c|c|c|c|c|c|}
\hline Polymer & $\begin{array}{c}\text { Number of } \\
-\mathrm{CH}_{2^{-}}\end{array}$ & $\begin{array}{c}\mathrm{E}_{\mathrm{a}}^{\beta_{1}} \\
\mathrm{~kJ} / \mathrm{mol}\end{array}$ & $\begin{array}{c}\mathrm{E}_{\mathrm{a}}^{\beta_{2}} \\
\mathrm{~kJ} / \mathrm{mol}\end{array}$ & $\mathrm{D}$ & $\begin{array}{c}\mathrm{T}_{0} \\
\mathrm{~K}\end{array}$ \\
\hline PET & 2 & 17 & 48 & 4.7 & 308 \\
\hline PTT & 3 & 23 & 49 & 5.2 & 272 \\
\hline PNT & $3^{*}$ & - & 49 & 6.2 & 290 \\
\hline PBT & 4 & 43 & 62 & 4.9 & 276 \\
\hline PPT & 5 & 32 & 47 & 6.4 & 240 \\
\hline
\end{tabular}

*For PNT this number refers to the following chemical structure of the glycolic subunit: $-\mathrm{CH}_{2}-\mathrm{CX}_{2}-\mathrm{CH}_{2}$, where $\mathrm{X}=-\mathrm{CH}_{3}$.

As one can see from the data collected in Table 1, PBT presents higher energy values for the two components of the $\beta$ process. This is probably due to the semicrystalline nature of the sample that cannot be quenched in the amorphous phase. As one can see, two different behaviours can be distinguished. The slower process $\left(\beta_{2}\right)$ presents essentially similar activation energy values for all the amorphous aromatic polyesters regardless of the methylene group number. On the contrary, the faster process $\left(\beta_{1}\right)$ 
seems to be dependent on the nature of the glycol moiety as far as the activation energy is concerned. Moreover, these effects can be reflected in the relaxation time activation plots shown in Figure 5. Here we have represented the relaxation times corresponding to the two components of the $\beta$ relaxation as a function of the reciprocal temperature for the different amorphous aromatic polyesters included in Table 1. PBT was omitted due to its semicristalline nature. It is clear that the data for the slower process $\left(\beta_{2}\right)$ almost lie on a similar line while a clear variation is observed for the faster $\beta_{1}$ process. In the inset of Figure 5 we plotted the activation energies of the two $\beta$ relaxation components as a function of the number of $-\mathrm{CH}_{2}$ - groups in the repetitive unit. Concerning the faster component $\left(\beta_{1}\right)$ of the $\beta$ relaxation, a dependence of $E_{\mathrm{a}}$ with the number of methylene groups is observed.

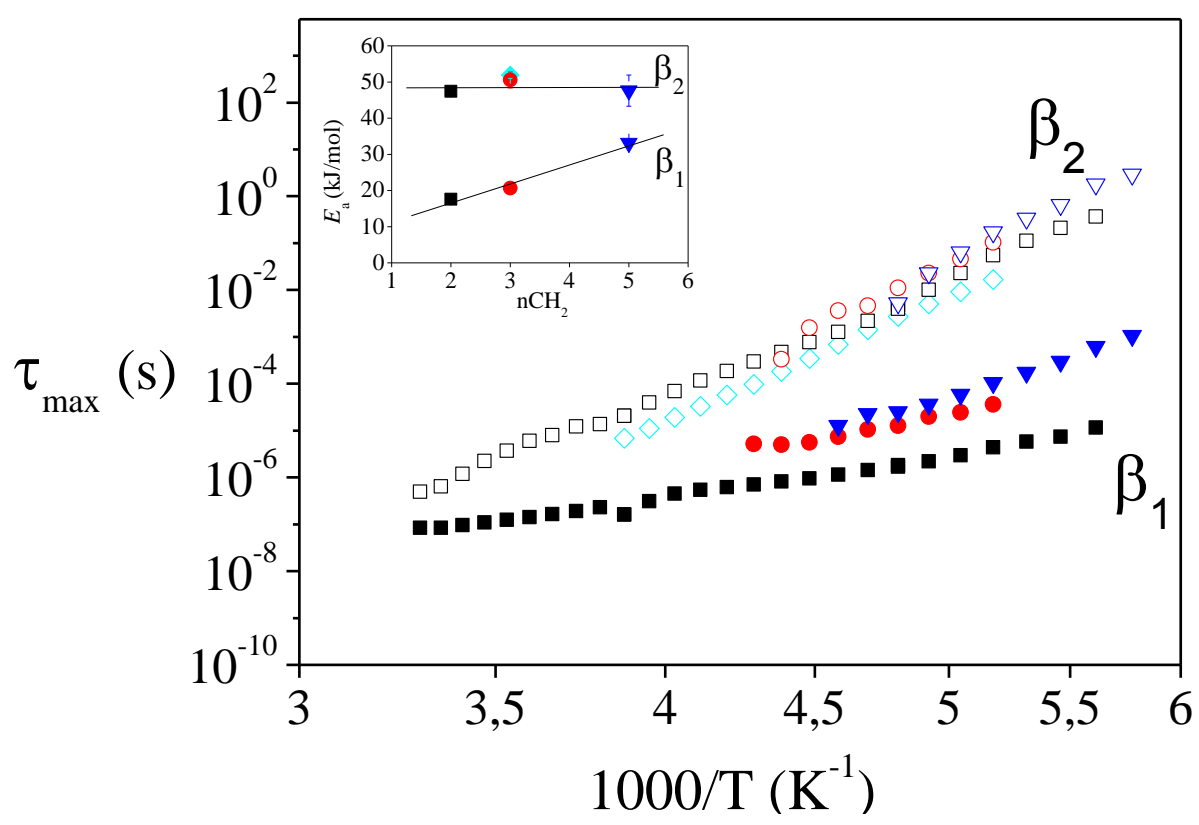

Figure 5. Average relaxation time as a function of reciprocal temperature of the $\beta$ relaxation components for different aromatic polyesters: $\beta_{1}$ solid symbols; $\beta_{2}$ open symbols. The inset shows the activation energy of the $\beta$ relaxation components as a function of the number of $\mathrm{CH}_{2^{-}}$groups in the repetitive unit of $\operatorname{PET}(\square), \operatorname{PTT}(\circ), \operatorname{PNT}(\diamond)$ and $\operatorname{PPT}(\boldsymbol{\nabla})$.

These results further support that $\beta_{1}$ can be related to the relaxation of $\mathrm{O}-\mathrm{C}$ bond and therefore is sensible to the distinct arrangement of the glycol moiety. Accordingly, the relaxation of longer glycol units is expected to overcome a higher energy barrier as compared to shorter ones. Moreover, this would explain the hindering of this mode in PNT, in which, the two methyl groups in the aliphatic subunit cause a steric hindrance that could make more difficult some conformations of the glycol unit. On the other hand, the activation energy calculated for the $\beta_{2}$ process keeps almost constant for all the aromatic polyesters. In this case no significant dependence of $E_{\mathrm{a}}$ on the glycol 
subunit length is observed. This fact supports that the molecular origin of the $\beta_{2}$ process can be related to the aliphatic CA-C bonds, since this bond does not changes by increasing the amount of $-\mathrm{CH}_{2}$ - units.

\subsubsection{The $\alpha$ relaxation of amorphous PPT}

Figure 6 shows the dielectric loss values at $30^{\circ} \mathrm{C}$ as a function of frequency for the amorphous PPT.

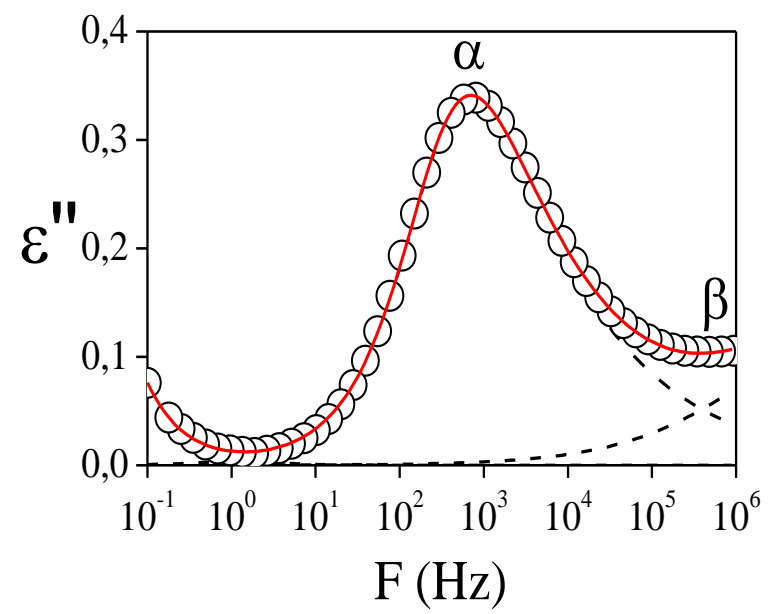

Figure 6. Dielectric loss values $\left(\varepsilon^{\prime \prime}\right)$ as a function of frequency, at $\mathrm{T}=30^{\circ} \mathrm{C}$, for amorphous PPT. Continuous line represents the best fit according to $\mathrm{HN}$ equation, dashed lines show the separated contribution of the different relaxation processes.

In the explored frequency window one can clearly see the $\alpha$ relaxation is accompanied at higher frequencies by the less intense contribution of the $\beta$ process and at low frequencies by a conductivity contribution. For fitting purposes, we consider here the $\beta$ process as a single relaxation since at high temperatures the two $\beta$ components tend to merge (Figure 3). In this case the dielectric loss spectrum can be described as a superposition of one $\mathrm{CC}$ function, accounting for the influence of the $\beta$ relaxation, an $\mathrm{HN}$ function related to the $\alpha$ relaxation and an additional term $-i\left(\sigma /\left(\varepsilon_{\mathrm{vac}} \omega\right)\right)^{s}$ describing the low frequency conductivity contribution. Here $\sigma$ is related to the direct current electrical conductivity, $\varepsilon_{\mathrm{vac}}$ is the dielectric constant of vacuum, and the value of the coefficient $0<s<1$ depends on the conduction mechanism ${ }^{9}$. The dashed lines in Figure 6 correspond to the contribution of the different processes and the continuous line is the total fit. The $\alpha$ relaxation of the amorphous polymer is characterized by a relatively narrow maximum in $\varepsilon^{\prime \prime}$ as a function of frequency with a symmetric broadening $b=0.8$ and an asymmetric broadening $c=0.5$. To estimate the relaxation time of maximum 
loss, $\tau_{\max }$, a strategy based on the Coburn and Boyd procedure ${ }^{10,27}$ was followed. The $\tau_{\max }$ values are shown in Figure 4 as a function of the reciprocal temperature. The $\tau_{\max }$ values of $\alpha$ relaxation exhibits a typical Vogel-Fulcher-Tamann (VFT) dependence described by:

$$
\tau_{\max }=\tau_{0} \exp \left(\frac{D T_{0}}{T-T_{0}}\right)
$$

where $\tau_{0}$ is a characteristic time, $T_{0}$ is the Vogel temperature, and $D$ is the fragility strength parameter ${ }^{9}$. This behaviour is characteristic of cooperative segmental motions appearing above the glass transition temperature. To obtain accurate fits, and in accordance with Angell's proposal ${ }^{34}$, a value of $\tau_{0}$ of $10^{-14} \mathrm{~s}$ was assumed. Continuous line in Figure 4 represents the best fit of the experimental $\tau_{\max }$ values to eq. 3 before crystallization occurs (up to about $20^{\circ} \mathrm{C}$ ). The corresponding parameters are collected in Table 1. For the sake of comparison, data for other aromatic polyesters have been included in this table. As one can see by comparing the results, the $T_{0}$ values, which are directly related to the glass transition temperature, decrease as the aliphatic methylene groups of the repetitive unit increase. This is expected on the basis of the progressive increasing of backbone flexibility as the glycol unit length increases. Although PNT seems to be an exception to this trend, being its $T_{0}$ value higher than PTT, one must consider the effect of the two side-chain methyls on the central carbon atom of the glycol unit. On the other hand, the $D$ parameter, which is inversely proportional to the fragility $^{35}$, increases with the methylene groups number evidencing that the larger is the aliphatic unit the lower is the fragility of the polymer. Nevertheless, looking at the PBT results $^{32}$, it is evident that crystallinity affects the $D$ and $T_{0}$ parameters, resulting in an extra lowering of the chain flexibility (higher $T_{0}$ values) accompanied by an increasing of the fragility (lower $D$ values).

\subsection{Isothermal cold-crystallization of PPT.}

Figure 7 shows the time-resolved dielectric loss spectroscopy spectra collected during the crystallization experiment at a crystallization temperature of $T_{\mathrm{c}}=30{ }^{\circ} \mathrm{C}$. The data have been normalized to the maximum at $t=0 \mathrm{~s}$. At this temperature the $\alpha$ relaxation is well resolved and clearly visible within the frequency range and the crystallization rate is slow enough to allow us to follow the process by DS in real time. As one can see, the initial amorphous state is characterized by an intense maximum in $\varepsilon^{\prime \prime}$ associated to the 
segmental dynamics. As the crystallization process takes place, the $\alpha$ relaxation suffers a significant decrease in intensity and the frequency of maximum loss shift towards lower values. In overall, this is the characteristic evolution of the $\alpha$ relaxation during crystallization as revealed by $\mathrm{DS}^{12,15}$.

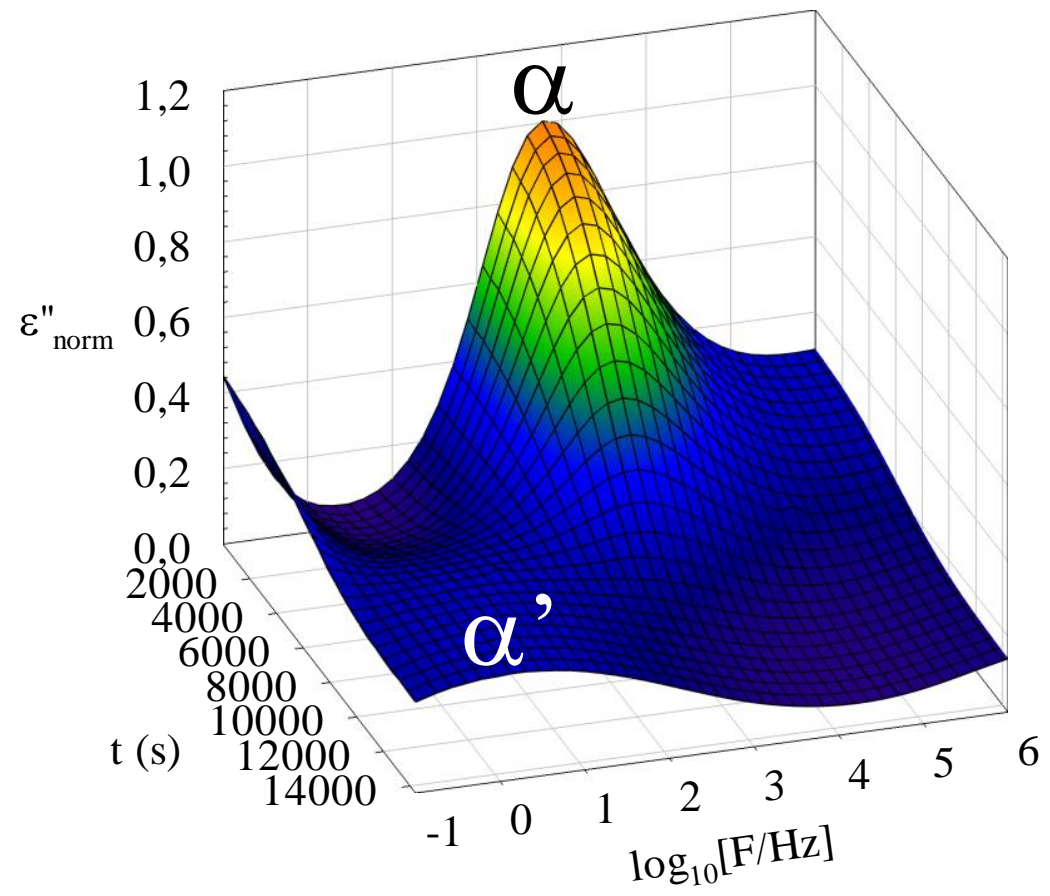

Figure 7. Time-resolved dielectric loss spectroscopy spectra collected during the crystallization experiment at a crystallization temperature of $T_{\mathrm{c}}=30^{\circ} \mathrm{C}$.

More precisely, Figure 8 shows the loss spectroscopy spectra collected for several characteristic crystallization times $t_{\mathrm{c}}$ during the crystallization experiment at $T_{\mathrm{c}}=30^{\circ} \mathrm{C}$. As one can see, the initial amorphous state (Figure $8 \mathrm{a}, t_{\mathrm{c}}=0 \mathrm{~s}$ ) is characterized by an intense maximum in $\varepsilon^{\prime \prime}$ associated to the segmental dynamics. In the initial stages of crystallization (Figure 8a) a small but detectable decrease of the $\alpha$ relaxation intensity is observed. As the crystallization process develops, the $\alpha$ relaxation suffers a significant modification consisting of the appearance of a new segmental process. This can be visualized in Figure $8 \mathrm{~b}\left(t_{\mathrm{c}}=8400 \mathrm{~s}\right)$. This effect, previously reported for $\mathrm{PET}^{11,36}$, can be described by an additional $\alpha$ process, labelled $\alpha^{\prime}$, appearing at lower frequency as crystallinity develops and associated to the segmental relaxation of a confined amorphous phase coexisting with the initial one. As crystallization further proceeds the $\alpha^{\prime}$ process becomes the main segmental process in the crystallized PPT sample. As an example, Figure $8 \mathrm{c}\left(t_{\mathrm{c}}=14400 \mathrm{~s}\right)$ shows the dielectric loss spectra of the crystallized sample. The dielectric data were analyzed, as described in the previous section, 
considering an additional $\mathrm{H}-\mathrm{N}$ contribution for the $\alpha^{\prime}$ process. The analysis of the experimental data is based on previous published works. In order the get a meaningful description of the evolution of the segmental relaxation with time during crystallization a model fitting for the $\alpha^{\prime}$ process has to be assumed. The model fitting is based on the known fact that the segmental relaxation of semicrystalline polymers is a symmetric process $^{11,12,20}$. Accordingly we can assume that since the $\alpha^{\prime}$ relaxation is the segmental motion restricted by the crystals it will have a $c=1$ value. The broadening parameter of the $\alpha^{\prime}$ process was fixed to the value obtained at the end of the crystallization when the segmental relaxation mostly consists on the $\alpha^{\prime}$ process. Good fits with physical meaning can be obtained then by letting the relaxation time of the $\alpha^{\prime}$ relaxation to slightly vary around the final value. In this way, estimates of the dielectric strength of the $\alpha^{\prime}$ process in addition those parameters of the $\alpha$ process can be obtained during the crystallization process. The results of the fittings are shown by the dotted lines.

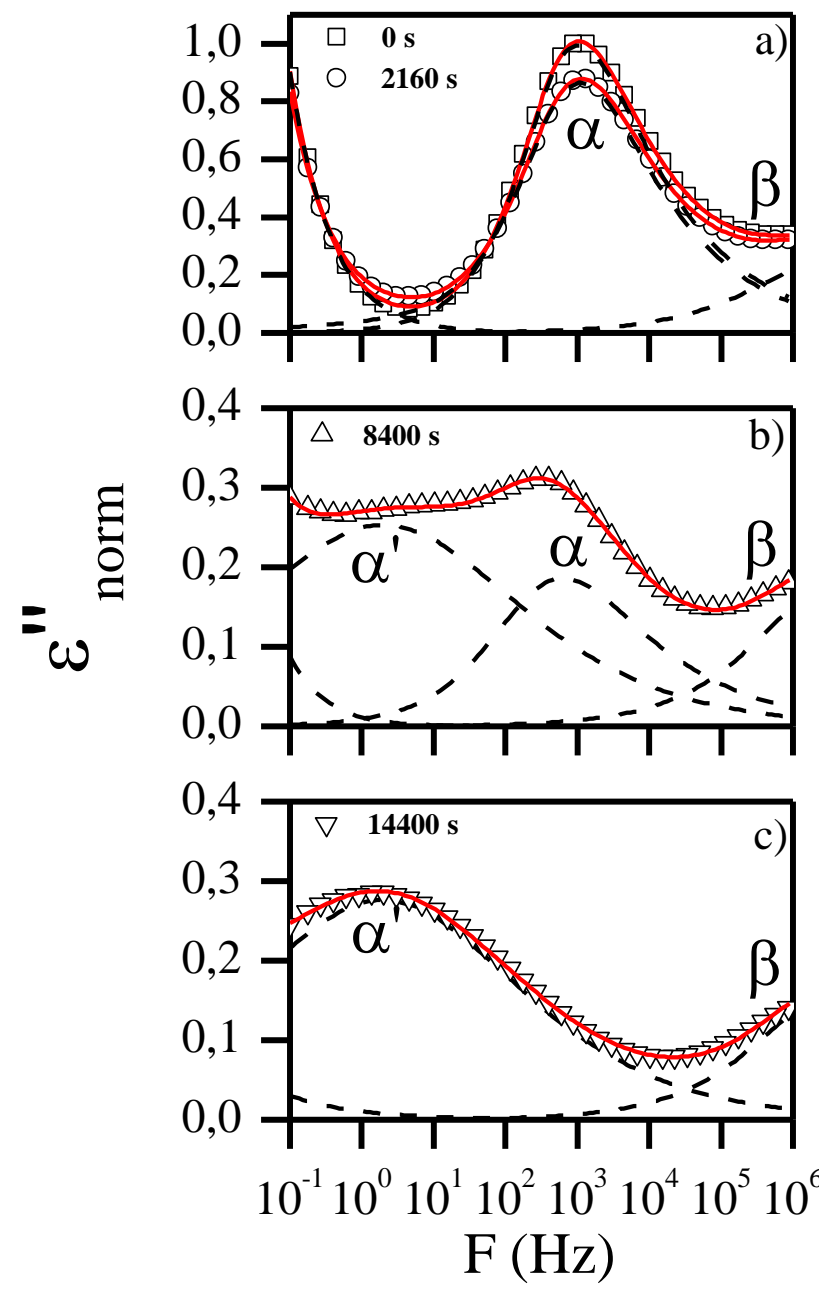

Figure 8. Isothermal $\varepsilon^{\prime \prime}$ data of initially amorphous PPT, at $\mathrm{T}_{\mathrm{c}}=30^{\circ} \mathrm{C}$, for different crystallization times. Continuous lines represent best fits according to $\mathrm{HN}$ equation, dashed lines show the separated contribution of the different relaxation processes and conductivity. 
Figure 9 represents the evolution with crystallization time, at $T_{\mathrm{c}}=30^{\circ} \mathrm{C}$, of the dielectric magnitudes. As one can see, as time increases the dielectric strength of the $\alpha$ relaxation decreases whereas the new process $\left(\alpha^{\prime}\right)$ grows up at the expense of the main $\alpha$ process. Crystallization affects not only the intensity of the dielectric relaxation but also its broadening and position. The $b$ and $c$ parameters decrease and increase, respectively. This means the $\alpha$ relaxation curve becomes broader and more symmetric. As expected, the $b$ parameter, connected with large scale motions ${ }^{37}$ is dramatically changed by crystallization. The $\alpha^{\prime}$ relaxation parameters $b, c$ and $\tau_{\max }$, have been considered to be constant during the whole crystallization process.

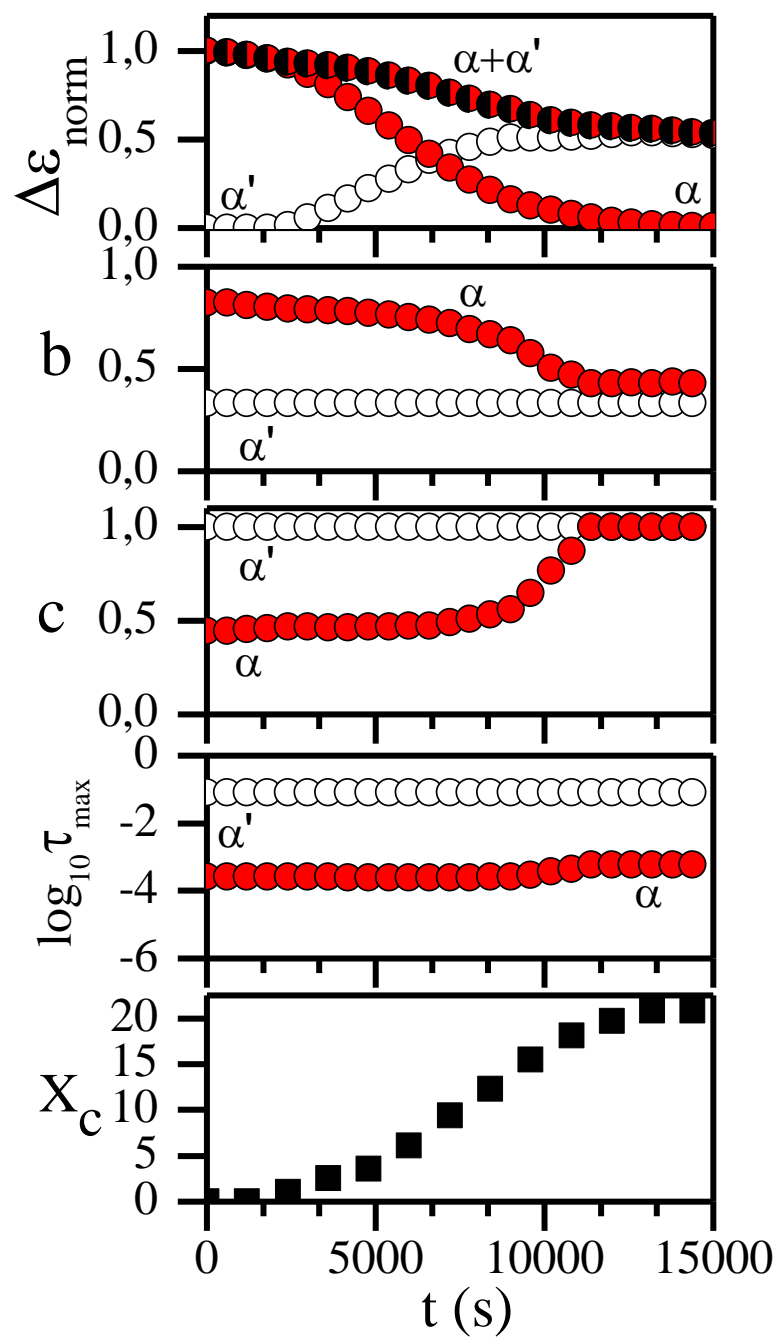

Figure 9. Havriliak-Negami parameters of the fitting of the $\alpha$ relaxation dielectric data of Figure 7 for the $\alpha(\bullet)$ and $\alpha^{\prime}(\circ)$ relaxations as a function of crystallization time: dielectric strength $(\Delta \varepsilon)$; shape parameters $(b$ and $c)$; time of maximum loss $\left(\log _{10} \tau_{\max }\right)$ and crystallinity degree $\left(X_{\mathrm{c}}\right)$. 
In order to study the influence of crystallinity on the $\beta$ relaxation we used a measuring protocol in which the crystallization process at $T_{\mathrm{c}}=30{ }^{\circ} \mathrm{C}$ was interrupted at selected times and the sample was cooled down to $\mathrm{T}=-85^{\circ} \mathrm{C}$ where a DS measurement of the $\beta$ relaxation was performed. Subsequently, the sample was heated up again to $T_{\mathrm{c}}=30^{\circ} \mathrm{C}$ and after the $\alpha$ relaxation observation the system was allowed to crystallize for a given period. By repeating this procedure several times the $\beta$ relaxation during the crystallization process was characterized. Figure 10 shows the dielectric loss spectra of the $\beta$ relaxation collected by the described procedure for some characteristic crystallization times during the crystallization experiment at $T_{\mathrm{c}}=30^{\circ} \mathrm{C}$. The continuous lines in Figure 10 represent the fits accomplished as previously described. The $\beta$ relaxation was considered as formed by the contribution of two symmetric CCprocesses during the whole crystallization process. In the early stage of crystallization, while the $\alpha$ relaxation clearly decreases in intensity with time (Figure 8a), the $\beta$ relaxations remain almost unaffected. As crystallization proceeds the main effect is a net decrease of the dielectric strength. As evidenced by the graphics in Figure 10, the $\beta_{1}$ component suffers a more intense decrease in the dielectric strength than the $\beta_{2}$ one. In particular, the $\Delta \varepsilon$ of the $\beta_{1}$ decreases by $18 \%$ with respect to its initial value, while the $\Delta \varepsilon$ of the $\beta_{2}$ just by $12 \%$. This result further supports the assignment of the $\beta_{1}$ component to the $\mathrm{O}-\mathrm{C}$ bond. In fact, this link is more mobile than the $\mathrm{CA}-\mathrm{C}$ one and, for this reason, could be more affected by the ordering of the chain during the development of the crystalline phase. Therefore, it is reasonable to think that the constriction due to crystallization may affect more the $\mathrm{O}-\mathrm{C}$ bond than the $\mathrm{CA}-\mathrm{C}$ one.

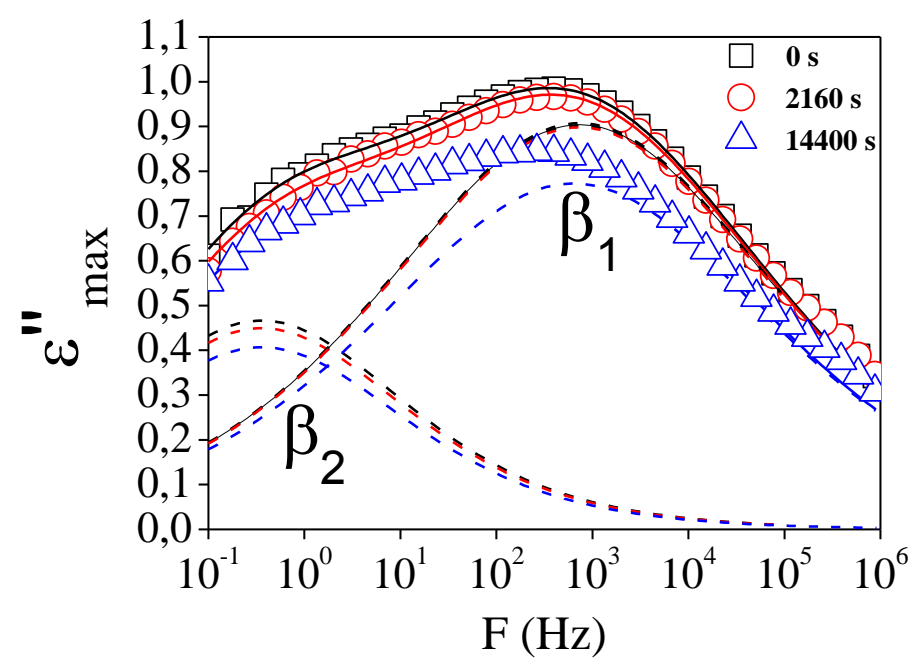

Figure 10. Dielectric loss as a function of frequency for the $\beta$ relaxation measured at $\mathrm{T}=-85^{\circ} \mathrm{C}$ for different crystallization times. Continuous lines represent best fits according to C-C equation, dashed lines show the separated contribution of the different relaxation processes. 
The study of the isothermal crystallization of PPT was also carried out by means of Wide-angle X-ray scattering (WAXS). Figure 11 shows the X-ray diffractograms of the isothermal cold-crystallized PPT sample. The Bragg maxima correspond to those reported for $\alpha$ phase with a triclinic unit cell of $\mathrm{PPT}^{2,6}$. The inset of Figure 11 shows some diffraction patterns obtained during the isothermal crystallization of PPT at $T_{\mathrm{c}}=$ $30{ }^{\circ} \mathrm{C}$, for different crystallization times. The times were chosen in order to be comparable with those of dielectric experiment (Figures 8 and 10). The occurrence of the crystallization process is revealed in the WAXS patterns by the onset of several Bragg reflections superimposed to the amorphous halo. An estimation of the fraction of crystalline phase $\left(X_{\mathrm{c}}\right)$ in the sample can be obtained by deconvoluting the Bragg reflections and the amorphous halo from the total reflections ${ }^{23,24}$.

Similarly to what happens to the $\beta$ relaxations, until crystallization times equal to 2400 seconds, no appreciable changes in the diffractograms are evidenced. The crystallinity degree $\left(X_{\mathrm{c}}\right)$ evolves with time in the characteristic sigmoidal shape represented in Figure 9 (bottom).

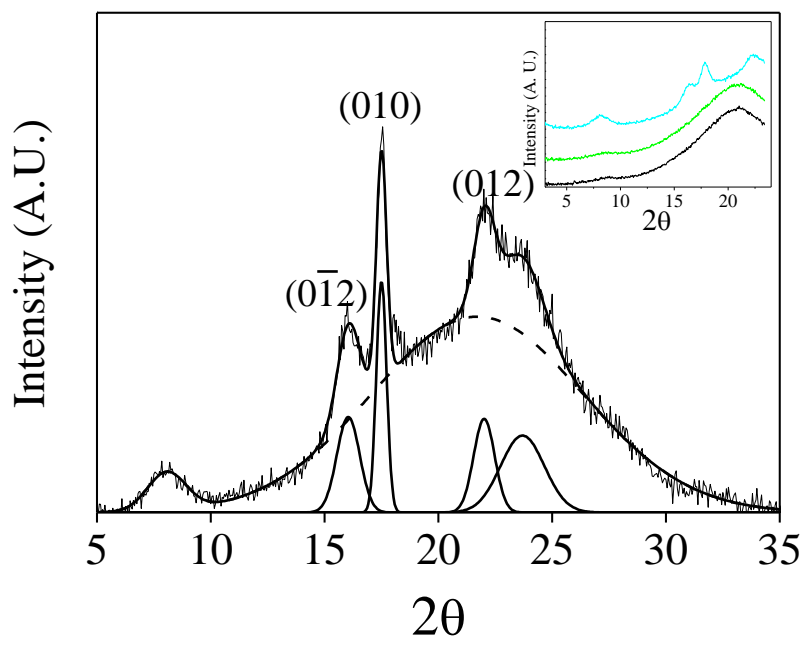

Figure 11. WAXS pattern of semicrystalline $\left(X_{\mathrm{c}}=20.8\right)$ PPT obtained by isothermal treatment at $\mathrm{T}=30^{\circ} \mathrm{C}$. The inset shows the WAXS diffracted intensity for PPT during the isothermal crystallization $\left(\mathrm{T}_{\mathrm{c}}=30{ }^{\circ} \mathrm{C}\right)$ at different crystallization times: $0 \mathrm{~s} ; 2400 \mathrm{~s}$ and $14400 \mathrm{~s}$ (from bottom to top).

Figure 12 represents for PPT the dependence of the total dielectric strength of $\alpha$ relaxation $\left(\Delta \varepsilon_{\alpha}+\Delta \varepsilon_{\alpha}\right)$ and $\beta$ process $\left(\Delta \varepsilon_{\beta 1}+\Delta \varepsilon_{\beta 2}\right)$, normalized with respect to their initial value, as a function of the crystallinity degree evaluated by WAXS $\left(X_{\mathrm{c}}\right)$. 


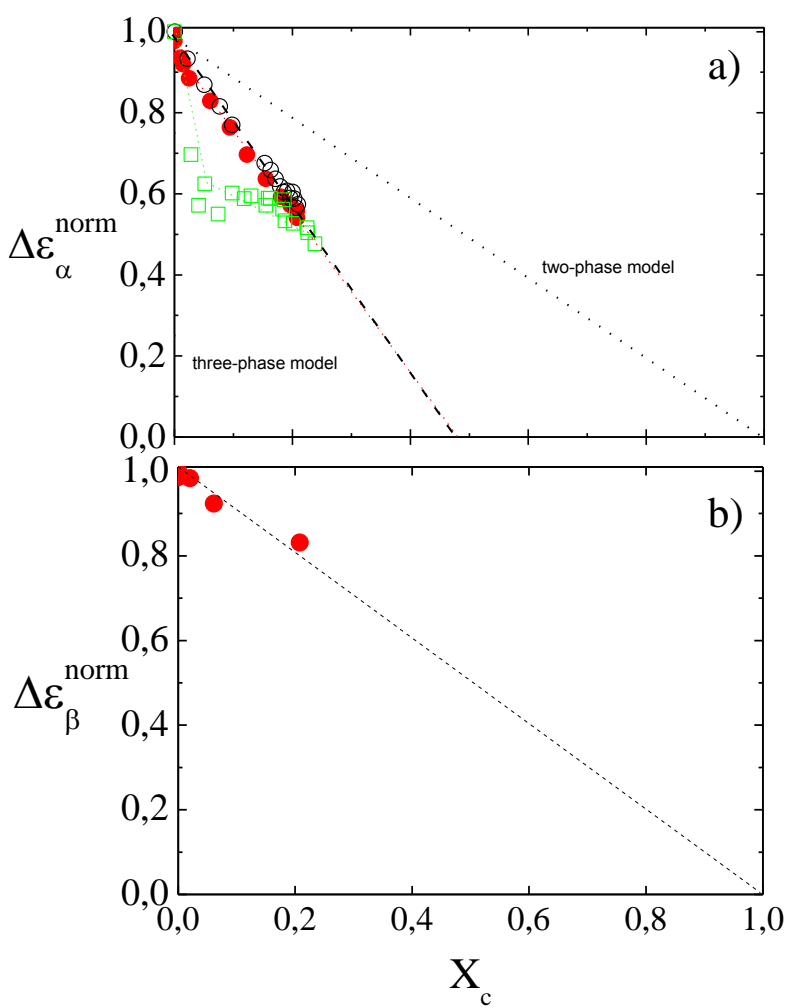

Figure 12. Normalized dielectric strength as a function of $X_{\mathrm{c}}$ for PPT (•). Data for PET $(\square)$ and PTT (०) are included for comparison ${ }^{20} . \Delta \varepsilon_{\alpha}^{n o r m}=\Delta \varepsilon_{\alpha}+\Delta \varepsilon_{\alpha^{\prime}}$ (top panel) and $\Delta \varepsilon_{\beta}^{n o r m}=$ $\Delta \varepsilon_{\beta 1}+\Delta \varepsilon_{\beta 2}$ (bottom panel). The dashed lines are a guide for the eye.

The dielectric strength of both $\alpha$ and $\beta$ relaxation decreases linearly with $X_{\mathrm{c}}$. However the slope for the $\alpha$ relaxation is significantly higher than that for the $\beta$ one. In a first approach, the dielectric strength is related to the amount of mobile amorphous phase while crystallinity relates to the amount of material included in the crystals. The results for the $\beta$ relaxation show a linear dependence with the crystallinity degree (Figure 12b). This fact indicates that the material incorporated into the crystalline phase is rather effectively arrested in its local dynamics while the whole amorphous phase can locally relax through the $\beta$ process. The same effect has been previously reported for $\operatorname{PET}^{10}$. As observed in Figure 12a, the decreases of $\Delta \varepsilon_{\alpha}^{n o r m}$ with $X_{\mathrm{c}}$ departs from the slope -1 which would be expected for a simple amorphous/crystal two phase model. This effect can be interpreted as an indication that, during isothermal crystallization, the immobilized polymer segments are not only those included in the crystals. On the contrary, there should be a significant fraction of non-relaxing and non-crystallized segments. As mentioned before, a common structural feature of polymers with a medium degree of crystallinity is the existence of a fraction of amorphous material, referred to as a rigid amorphous phase (RAP), which cannot relax at the same rate as the 
main amorphous phase ${ }^{16,17}$. Moreover, the dielectric strength for PPT decreases lineally with $X_{\mathrm{c}}$ with the same slope for the whole range suggesting that the rate of reduction of relaxing species is almost constant during crystallization. This behaviour is similar to that found for PTT ${ }^{20}$. However, similar studies on PET have shown that the dependence of $\Delta \varepsilon_{\alpha}^{n o r m}$ with $X_{\mathrm{c}}$ changes the slope drastically from values lower than -1 to values close to $-1^{20}$. Recently a similar behaviour has been reported for poly(lactide $)^{38}$. For the sake of comparison the results for two other terephthalic acid containing polyesters, PET $^{11}$ and PTT $^{20}$ have been included in Figure 12a. In order to explain this effect it was proposed that cold crystallization of PET proceeds by filling the space with a heterogeneous distribution of stacks of lamellae separated by liquid pockets ${ }^{11}$. In this case, the RAP is mostly located in the inter-lamellar amorphous regions, being the material in the liquid pockets (inter-lamellar stacks amorphous regions) the main relaxing species giving rise to the dielectric segmental relaxation. In this view, the initial strong decrease of $\Delta \varepsilon_{\alpha}^{\text {norm }}$ with $X_{\mathrm{c}}$ can be associated to the immobilization of PET segments in the intra-lamellar stacks amorphous regions while the second weaker dependence can be associated to the formation of secondary lamellar stacks in the liquid pockets $^{11}$. This scenario was also proposed for poly(butylene isophthalate) ${ }^{39}$. For PTT, and PPT the reduction of $\Delta \varepsilon_{\alpha}^{\text {norm }}$ with $X_{\mathrm{c}}$ is weaker than in the previous cases and exhibits a single regime. For PTT this effect has been interpreted considering a homogeneous filling of the space by uniformly separated crystalline lamellae rendering to an absence of liquid pockets. In this case the RAP should be associated to the crystalline-amorphous interface rather than to the whole inter-lamellar amorphous regions $^{20}$. Morphological ${ }^{40}$ and dielectric ${ }^{20}$ studies on PTT indeed suggested a quite homogeneous crystal lamellae filling. For PPT the scenario is clearly closer to that of PTT rather than to that of PET. This suggests for PPT a rather homogeneous filling of the space by uniformly separated crystalline lamellae. Morphological studies on cold and melt crystallized PPT reported on the formation of large and homogeneous spherulites as for the PTT case $^{41}$. For polyesters of the type n-glycol terephthalate (nGT) crystal structures determination have shown that 2GT (PET) crystallizes with the chain within the unit cell in a fully extended conformation while 3GT(PTT), 4GT(PBT) and 5GT(PPT) all crystallize with the chain contracted ${ }^{2}$. In our case we can propose that data shown in Figure 12a indicate that these conformational differences of the crystals may affect the homogeneity of the lamellar crystal distribution. 


\section{Conclusions}

To sum up, the sub-glass dynamics of PPT as revealed by dielectric spectroscopy, is characterized by the existence of two processes, $\beta_{1}$ and $\beta_{2}$, which have been assigned to the relaxation of the bond between the ester oxygen and the aliphatic carbon of the glycol subunit, and to the bond between the aromatic ring carbon to the ester carbon, respectively. The comparison with the sub-glass dynamics of other closely related aromatic polyesters like PET and PTT, evidenced that the faster component $\beta_{1}$ strongly depends on the amount of methylene groups of the monomer, $\beta_{2}$ is not considerably affected by the nature of the glycol subunit. During cold crystallization, the dielectric strength of $\beta$ relaxation linearly decreases indicating that the material incorporated into the crystalline phase is rather effectively arrested in its local dynamics. The $\alpha$ relaxation suffers a significant modification consisting of the appearance of a new segmental process $\left(\alpha^{\prime}\right)$ associated to the segmental relaxation of a confined amorphous phase coexisting with the initial one. The evolution of the $\alpha$ process suggest that for PPT the crystal lamellar stacks are homogeneously distributed, lacking of broad amorphous domains, liquid pockets, as observed for other similar polyesters.

\section{Acknowledgments}

Financial support by MAT2008-03232, MAT2011-23455, and MAT2012-33517 from MINECO is gratefully acknowledged. M.S. thanks CSIC and the Fondo Social Europeo (FSE) for cofinancing the JAE-Doc contract.

\section{References}

1. J. Schultz and J. Wu, edited by S. Fakirov (Wilwy-VCH Verlach, Weinheim, 2002), Vol. 1, pp. 551-573.

2. I. H. Hall, M. G. Pass and N. N. Rammo, Journal of Polymer Science Part BPolymer Physics 16 (8), 1409-1418 (1978).

3. I. Goodman, Angewandte Chemie-International Edition 74 (16), 606-\& (1962).

4. L. W. Pi, E. M. Woo and H. L. Liu, Journal of Polymer Science Part B-Polymer Physics 42 (23), 4421-4432 (2004).

5. P. L. Wu and E. M. Woo, Journal of Polymer Science Part B-Polymer Physics 42 (7), 1265-1274 (2004).

6. I. H. Hall and N. N. Rammo, Journal of Polymer Science Part B-Polymer Physics 16 (12), 2189-2214 (1978).

7. McCrum N.G., Read B.E. and W. G., Anelastic and Dielectric Effects in Polymeric Solids. (Wiley, London, 1967).

8. G. R. Strobl, The Physics of Polymers. ( Springer, Berlin, Germany, 2003).

9. F. Kremer and A. Schonhals, (Springer Verlag, Heidelberg, Germany, 2002).

10. J. C. Coburn and R. H. Boyd, Macromolecules 19 (8), 2238-2245 (1986). 
11. C. Alvarez, I. Sics, A. Nogales, Z. Denchev, S. S. Funari and T. A. Ezquerra, Polymer 45 (11), 3953-3959 (2004).

12. T. A. Ezquerra, J. Majszczyk, F. J. Baltacalleja, E. Lopezcabarcos, K. H. Gardner and B. S. Hsiao, Physical Review B 50 (9), 6023-6031 (1994).

13. M. Soccio, A. Nogales, N. Lotti, A. Munari and T. A. Ezquerra, Physical Review Letters 98 (3) (2007).

14. M. Soccio, A. Nogales, N. Lotti, A. Munari and T. A. Ezquerra, Polymer 48 (16), 4742-4750 (2007).

15. A. R. Bras, P. Malik, M. Dionisio and J. F. Mano, Macromolecules 41 (17), 6419-6430 (2008).

16. P. T. Huo and P. Cebe, Macromolecules 25 (2), 902-909 (1992).

17. M. Pyda, E. Nowak-Pyda, J. Mays and B. Wunderlich, Journal of Polymer

Science Part B-Polymer Physics 42 (23), 4401-4411 (2004).

18. J. Dobbertin, A. Hensel and C. Schick, Journal of Thermal Analysis 47 (4), 1027-1040 (1996).

19. S. Kalakkunnath and D. S. Kalika, Polymer 47 (20), 7085-7094 (2006).

20. A. Sanz, A. Nogales, T. A. Ezquerra, M. Soccio, A. Munari and N. Lotti, Macromolecules 43 (2), 671-679 (2010).

21. M. Soccio, N. Lotti, L. Finelli, M. Gazzano and A. Munari, Journal of Polymer Science Part B-Polymer Physics 46 (2), 170-181 (2008).

22. I. Šics, T. A. Ezquerra, A. Nogales, F. J. Baltá-Calleja, M. Kalniņš and V. Tupureina, Biomacromolecules 2 (2), 581-587 (2001).

23. D. J. Blundell and B. N. Osborn, Polymer 24 (8), 953-958 (1983).

24. Z. Denchev, A. Nogales, T. A. Ezquerra, J. Fernandes-Nascimento and F. J.

Balta-Calleja, Journal of Polymer Science Part B-Polymer Physics 38 (9), 1167-1182 (2000).

25. Havriliak.S and S. Negami, Polymer 8 (4), 161-\& (1967).

26. S. U. Boyd and R. H. Boyd, Macromolecules 34 (20), 7219-7229 (2001).

27. A. Nogales, Z. Denchev, I. Sics and T. A. Ezquerra, Macromolecules 33 (25), 9367-9375 (2000).

28. S. P. Bravard and R. H. Boyd, Macromolecules 36 (3), 741-748 (2003).

29. M. Soccio, A. Nogales, M. C. Garcia-Gutierrez, N. Lotti, A. Munari and T. A. Ezquerra, Macromolecules 41 (7), 2651-2655 (2008).

30. C. R. Ashcraft and R. H. Boyd, Journal of Polymer Science Part B-Polymer Physics 14 (12), 2153-2193 (1976).

31. M. Soccio, A. Nogales, T. A. Ezquerra, N. Lotti and A. Munari, Macromolecules 45 (1), 180-188 (2012).

32. A. Sanz, A. Nogales, N. Lotti, A. Munari and T. A. Ezquerra, Journal of NonCrystalline Solids 353 (41-43), 3989-3995 (2007).

33. A. Nogales, A. Sanz and T. A. Ezquerra, Journal of Non-Crystalline Solids 352 (42-49), 4649-4655 (2006).

34. C. A. Angell, Polymer 38 (26), 6261-6266 (1997).

35. R. Bohmer, K. L. Ngai, C. A. Angell and D. J. Plazek, Journal of Chemical Physics 99 (5), 4201-4209 (1993).

36. K. Fukao and Y. Miyamoto, Physical Review Letters 79 (23), 4613-4616 (1997).

37. A. Schonhals and E. Schlosser, Colloid and Polymer Science 267 (2), 125-132 (1989).

38. E. Laredo, M. Grimau, A. Bello and D. Wu, European Polymer Journal 49 (12), 4008-4019 (2013). 
39. A. Sanz, A. Nogales, T. A. Ezquerra, N. Lotti, A. Munari and S. S. Funari, Polymer 47 (4), 1281-1290 (2006).

40. D. A. Ivanov, G. Bar, M. Dosiere and M. H. J. Koch, Macromolecules 41 (23), 9224-9233 (2008).

41. P. L. Wu, E. M. Woo and H. L. Liu, Journal of Polymer Science Part B-Polymer Physics 42 (23), 4421-4432 (2004). 